\title{
Über einen interessanten Fall von Brumnen-Verunreinigungen.
}

\author{
Von
}

\author{
Dr. A. Bömer in Münster i. W.
}

Im Anschlusse an das Referat des Herrn Geheimrat König über den gegenwärtigen Stand der Beurteilung von Trink- und Abwasser nach der chemischen Analyse möchte ich hier einen interessanten Fall von Brunnen-Verunreinigungen kurz beschreiben, den wir im Frühjahre des vergangenen Jahres zu beobachten Gelegenheit hatten.

Die Besitzer der Brunnen II, III und IV zu G. in Westfalen (vergl, die Skizze S. 88) klagten gegen die Kohlenzeche M. wegen Verunreinigung ihrer Brunnen durch die Abwässer der Zeche. Der Besitzer des Brunnens I war von der Zeche bereits für die Verunreinigung seines Brunnens entschädigt worden. Im Auftrage des Landgerichtes zu E. sollten wir infolgedessen ein Gutachten darüber abgeben, ob die Brunnen II, III und IV durch die Abwässer der beklagten Zeche oder - wie die Beklagte behauptete - durch Zuflüsse aus den Aborten und Stallungen der Brunnenbesitzer unbrauchbar gemacht seien.

Bei den Brunnen I, II und III handelte es sich um Bohrbrunnen, welche in ihrem oberen Teile, soweit das Erdreich locker war, ausgemauert waren, während in den unterliegenden Mergelschichten das Bohrloch vollständig frei $d$. h. ohne jeglichen seitlichen Abschluß war, so daß aus den mehr oder minder zerklüfteten Mergelschichten ein reichlicher seitlicher WasserzufluB erfolgen konnte. Die Tiefe der Brunnen I und III betrug 16-17 $\mathrm{m}$ und die des Brunnens II $20 \mathrm{~m}$. Der Brunnen IV, auf einem kleinen Gehöft gelegen, war ein gewöhnlicher Kesselbrunnen von geringer Tiefe, der durch eine Steinplatte abgedeckt war.

Aus allen vier Brunnen wurde eine Probe des Wassers entnommen. Um einen Anhalt für die Zusammensetzung reiner Brunnenwässer der betreffenden Gegend zu erhalten, wurde auch das Wasser zweier in der Nähe gelegenen, angeblich nicht verunreinigten Brunnen untersucht, von denen der Brunnen $V$, südlich der verunreinigten Brunnen gelegen, ebenfalls ein Bohrbrunnen war, während der Brunnen VI, nordwestlich der verunreinigten Brunnen gelegen, ein gewöhnlicher Kesselbrunnen war.

Die Abwässer der beklagten Zeche bestanden zunächst aus den Grubenwässern, welche am Nordrande des Zechenplatzes der Zechenbahn entlang, zunächst in einem geschlossenen Kanale, dann vom Punkte a an oberirdisch, in ostwestlicher Richtung abgeführt wurden. Bis einige Zeit vor der Probenahme waren dieselben in einem offenen Graben abgeführt worden, später, nachdem sich die Verunreinigung des Brunnens I gezeigt hatte, war der offene Graben im westlichen, diesem Brunnen zunächst gelegenen Teile des Zechengrundstückes durch einen geschlossenen, gemanerten Kanal ersetzt worden; kurz vor der Probenahme war auch der noch übrige Teil des Grabens durch einen gemauerten offenen Kanal ersetzt worden. Beim Einfluß des Wassers aus diesem offenen in den geschlossenen Kanal, beim Punkte A der Skizze, wurde eine Probe des Grubenwassers entnommen. 
Der südliche Rand des durch Anschüttungen erhöhten Zechenplatzes fällt nach dem sich anschließenden Wiesengelände mit einer steilen Böschung ab. Am Fuße der Böschung trat bei b ein Kanal aus, dessen Wasser aus dem Sickerwasser des Zechenplatzes einschlieblich der von der Kohlenwäsche und der Kokslöschung abfließenden Wässer bestand. Der Kanalausfluß mündete in einen südlich des Zechenplatzes in ostwestlicher Richtung führenden offenen Graben, der zur Zeit der Probenahme infolge der an den voraufgehenden Tagen gefallenen reichlichen Regenmengen noch verschiedene Zuflüsse aus den anliegenden Wiesen er-

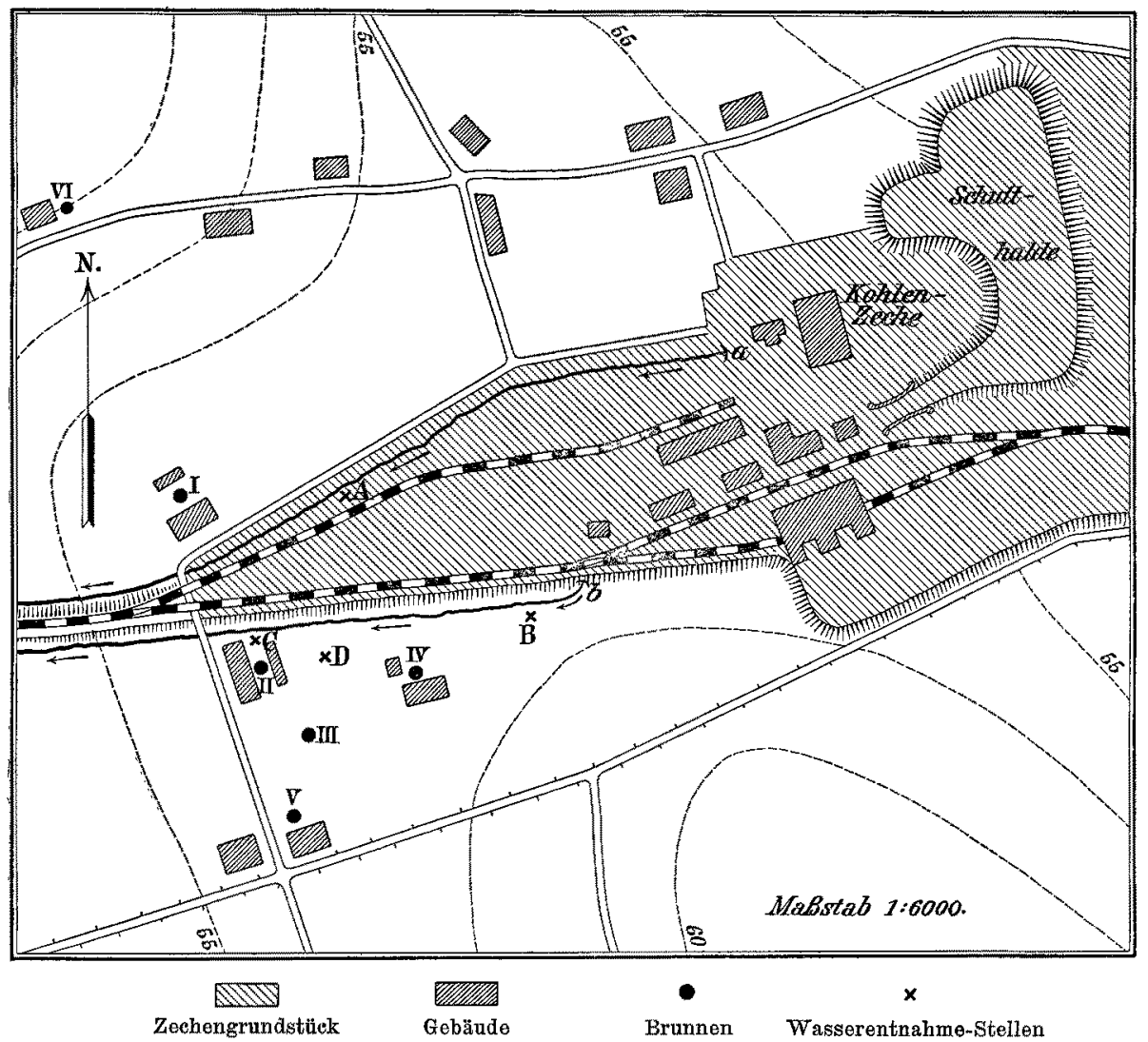

Fig. 1. Skizze der Örtlichkeit.

hielt. Von den Wässern dieses offenen Grabens entnahm ich eine Probe bei B, unmittelbar beim Austritt des Wassers aus dem Zechengrundsticke, und eine zweite bei $\mathrm{C}$ in der Höhe des verunreinigten Brunnens II. Endlich wurde auch noch aus dem Garten östlich des Brunnens II bei Punkt D eine Probe des Grundwassers entrommen, welches sich in einem zu diesem Zwecke ausgeworfenen Loche in kurzer Zeit angesammelt hatte und bis etwa $3 / 4 \mathrm{~m}$ unter Terrain angestiegen war.

Die Lagenverhältnisse der Örtlichkeit sind aus obenstehender Skizze ersichtlich. Die Untersuchung der entnommenen Wasserproben lieferte folgende Ergebnisse: 


\begin{tabular}{|c|c|c|c|c|c|c|c|c|c|c|c|c|c|c|}
\hline \multirow[b]{2}{*}{ No. } & \multirow{2}{*}{\multicolumn{2}{|c|}{$\begin{array}{l}\text { Bezeichnung } \\
\text { der } \\
\text { Wasserproben }\end{array}$}} & \multirow[b]{2}{*}{$\begin{array}{c}\text { Aussehen } \\
\text { des } \\
\text { Wassers }\end{array}$} & \multicolumn{9}{|c|}{1 Liter Wasser enthielt mg } & \multirow[b]{2}{*}{ 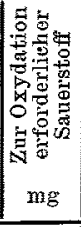 } & \multirow[b]{2}{*}{ 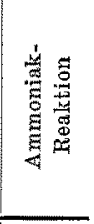 } \\
\hline & & & & 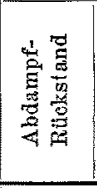 & 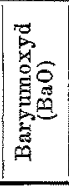 & 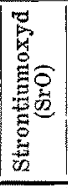 & 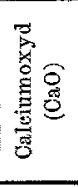 & 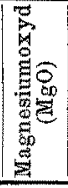 & 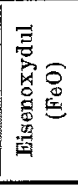 & 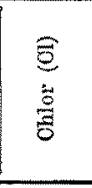 & 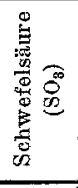 & 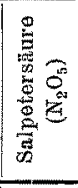 & & \\
\hline 1 & \multirow{7}{*}{$\begin{array}{c}\text { Brannen- } \\
\text { wässer }\end{array}$} & $\mathrm{I}$ & ( bräunlich) & 62020,0 & 12,0 & 20,0 & 6181,5 & 416,3 & 1791,0 & 32482,5 & 130,4 & 30,4 & 140,8 & stark \\
\hline 2 & & $\mathrm{II}$ & ( trübe $\}$ & 8620,0 & 15,0 & 15,0 & 1391,5 & 25,2 & 256,5 & 2534,7 & 1581,4 & 30,4 & 8,2 & schwach \\
\hline 3 & & III & $\left\{\begin{array}{c}\text { schwach } \\
\text { gelblieh } \\
\text { tritbe }\end{array}\right\}$ & 4520,0 & 15,0 & 20,0 & 980,2 & 18,0 & 13,5 & 1370,3 & 103,0 & 3,0 & 5,5 & 0 \\
\hline 4 & & IV & \{ hell u. o a & 1530,0 & - & - & 360,0 & 27,0 & - & 170,4 & 278,2 & 173,3 & 3,9 & 0 \\
\hline $\mathbf{5}$ & & & lklar $\mathrm{b}$ & $(\mathbf{1 1 8 4 , 0 )}$ & - & - & $(332,5)$ & $\cdots$ & - & $(177,0)$ & $(259,3)$ & $(130,1)$ & $(5,9)$ & $(0)$ \\
\hline 6 & & $\mathrm{~V}$ & $\left\{\begin{array}{c}\text { holl, } \\
\text { sehwaeh }\end{array}\right.$ & 660,0 & - & - & 260,0 & 10,8 & 一 & 85,2 & 138,9 & 30,4 & 1,5 & 0 \\
\hline 7 & & $\mathrm{VI}$ & $\left(\begin{array}{c}\text { opali- } \\
\text { siorend }\end{array}\right\}$ & 460,0 & 一 & - & 185,0 & 18,0 & - & 28,4 & 51,5 & 6,1 & 3,6 & 0 \\
\hline 8 & \multirow{3}{*}{\multicolumn{2}{|c|}{ 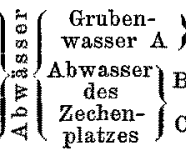 }} & $\left\{\begin{array}{c}\text { bräunlich } \\
\text { trübe }\end{array}\right\}$ & 118500,0 & 718,8 & 810,0 & 5371,5 & 887,4 & 202,5 & 59569,0 & 14,6 & 39,5 & 36,0 & stark \\
\hline 9 & & & hell u. klar & 1535,0 & - & - & 340,0 & 68,4 & - & 134,9 & 566,6 & 66,9 & 4,1 & dentlieh \\
\hline 10 & & & $\left\{\begin{array}{c}\text { sehwaoh } \\
\text { getribt }\end{array}\right\}$ & 600,0 & - & - & 185,0 & 30,6 & - & 49,7 & 242,1 & 21,3 & 4,2 & Spur \\
\hline 11 & $\underset{D}{G r u n d w a}$ & & $\left\{\begin{array}{c}\text { schwach } \\
\text { gelblich }\end{array}\right\}$ & 970,0 & - & - & 170,0 & 43,3 & - & 85.2 & 223,1 & 152,0 & 12,5 & 0 \\
\hline
\end{tabular}

Anmerkungen zar Tabelle.

1. Keines der Wässer enthielt Salpetrige Säure.

2. Das Grubenwasser A enthielt $60,0 \mathrm{mg}$ vorwiegend aus Eisenverbindungen bestehende Schwebestofte.

3. Die für das Brunnenwasser IV unter $b$ angegebenen Zahlen waren von uns im Jahre 1896 ermittelt worden.

4. Bei denjenigen Wässern, bei denen eine Zahl für den Gehalt an Baryumoxyd, Strontiumoxyd und Eisenoxydul nicht angegeben ist, sind diese Verbindungen nicht bestimmt worden.

Betrachten wir zunächst die Abwässer der Zeche, so haben wir in dem Grubenwasser A einen ausgesprochenen Vertreter der im rheinisch-westfälischen Kohlenbezirk nicht selten vorkommenden Grubenwässer vor uns, welche neben Chloriden der Alkalien, des Calciums und des Magnesiums noch einen hohen Gehalt an Chloriden des Baryums und Strontiums besitzen. Diese Art von Grubenwässern fällt bei der gewöhnlichen Analyse sofort durch ihren naturgemäß nur geringen Gehalt an Schwefelsäure auf, von der die große Mehrzahl der kochsalzreichen Wässer der Kohlengruben sonst in der Regel beträchtliche Mengen enthalten,

Das Abwasser des Zechenplatzes B, wie es aus der Böschung des Zechenplatzes ausfloß, dagegen war gekennzeichnet durch seinen hohen Gehalt an Calciumsulfat, während der Gehalt an Chloriden nur ein mäßig hoher war. Der westliche Teil des Zechenplatzes, über den die Zechenbahn führt, besteht, wie schon angedeutet wurde, aus einer Anschüttung aus dem Abraum-Gestein der Grube. Dieses Gestein besteht im rheinisch-westfälischen Kohlengebirge aus einem mehr oder minder kalkreichen grauen bis schwarzen Kohlenschiefer, der durehweg nicht unbeträchtliche Mengen von Schwefelkies enthält. 
Wird dieser Kohlenschiefer zutage gefördert und hier der Finwirkung von Luft und Regen ausgesetzt, so zerfällt er sebr bald und der darin vorhandene Schwefelkies setzt sich dabei nach der Formel

$$
\mathrm{FeS}_{2}+7 \mathrm{O}+\mathrm{H}_{2} \mathrm{O}=\mathrm{FeSO}_{4}+\mathrm{H}_{2} \mathrm{SO}_{4}
$$

zu Ferrosulfat und freier Schwefelsäure um. Findet die letztere in dem Gestein hinreichende Mengen Basen, vor allem Kalk, so wird sie durch diese gebunden. Sind aber genügende Mengen von Basen nicht vorhanden, so erscheint die Schwefelsäure nicht selten in den Sickerwässern der Schutthalden neben mehr oder minder beträchtlichen Mengen von Ferrosulfat im freien Zustande. Hieraus erklärt sich der hohe Gehalt des Abwassers B an Sulfaten.

Das Abwasser $\mathrm{C}$ erwies sich qualitativ ähnlich zusammengesetzt wie $\mathrm{B}$, nur war es infolge der Zuflüsse von Tageswässern aus den anliegenden Wiesen wesentlich verdünnter.

Gehen wir nunmehr zur Betrachtung der verunreinigten Brunnenwässer über, so sehen wir, daß das Brunnenwasser I ungemein reich an Salzen ist es enthält über $62 \mathrm{~g}$ Trockenrückstand in 1 Liter -, unter denen neben großen Mengen von Chlornatrium vorwiegend die Chloride des Calciums, Magnesiums und Eisens sich finden. Dagegen ist der Gehalt an Schwefelsäure nur ein verhältnismäßig geringer. Nach diesen Untersuchungsergebnissen war es zweifellos, daß der Brunnen I in außerordentlich starkem Maße, durch das in etwa $35 \mathrm{~m}$ Entfernung - bis vor einiger Zeit in einem offenen Graben in angeschüttetem und daher lockerem Boden - vorbeifließende Grubenwasser der Zeche verunreinigt war. Auffallend erscheint nur im ersten Augenblicke, daß bei dem hohen Salzgehalt des Brunnenwassers sein Gehalt an Baryum und Strontium, welche in dem Grubenwasser in so verhältnismäßig großer Menge vorhanden waren, nur gering ist. Andererseits sehen wir aber auch, daß der Schwefelsäuregehalt des verunreinigten Brumnenwassers rund neunmal so hoch ist als der des Grubenwassers. Auf Grund der vorherigen Ausführungen ergibt sich nun leicht, daß diese Umsetzungen durch das Zusammentreffen des Chlorbaryum und Chlorstrontium enthaltenden Grubenwassers mit den schwefelsäurereichen Sickerwässern der Anschüttungen bezw. dem durch diese schon mit Sulfaten angereicherten Grundwasser verursacht sein müssen. Der Brunnen war schon seit dem Jahre 1896 verunreinigt und daher außer Betrieb gesetzt.

Ähnlich lagen die Verhältnisse bei den verunreinigten Brunnen II und III. Diese waren von dem Grubenwasser-Kanale bezw. -Graben etwa 70 bezw. $120 \mathrm{~m}$, also viel weiter entfernt, als der Brunnen I; ferner lag zwischen dem Brunnen II und III und dem Grubenwasser-Kanal eine weit größere Anschüttung als bei dem Brunnen I und endlich floß zwischen dieser und den beiden Brunnen II und III auch der südliche offene Graben mit den Wässern $B$ und $C$ hindurch. Es ist daher leicht erklärlich, daß der Schwefelsäuregehalt der Wässer vor allem bei dem Brunnen II ein weit höherer war. Trotzdem fauden sich auch in diesem Wasser geringe Mengen Baryum und Strontium und es war daher der Brunnen II sowohl durch Gruben. wasser wie durch das Sickerwasser der Anschüttungen verunreinigt.

Der Brunjen III lag noch wesentlich weiter (etwa $50 \mathrm{~m}$ weiter) von dem Grubenwasser-Graben entfernt als der Brunnen II, aber auch bei ihm ist aus dem hohen Gehalte an Calciumoxyd und Chlor auf eine Verunreinigung durch das Grubenwasser zu schlieben; dagegen ist bei dem Brumen III die Zuführung von Sickerwasser aus den Anschüttungen, wie aus dem nur mäßig hohen Schwefelsäuregehalte zu schließen 
ist, anscheinend nicht so bedeutend gewesen, wie bei dem Brunnen II. Diese verschiedene Zusammensetzung der Wässer der beiden Brunnen II und III kann bei der bekanntermaßen sehr häufig unregelmäßigen Wasserführung in Mergelschichten kaum befremden. Es mag auch sein, daß die beiden Brunnen zu verschiedenen Zeiten verunreinigt worden waren und die Zusammensetzung sowohl des Grubenwassers je nach der Grubensoble, von welcher gepumpt wurde, als auch der Sickerwässer der Anschüttungen je nach den Witterungsverhältnissen eine verschiedene gewesen war.

Jedenfalls komnte nach der chemischen Zusammensetzung der Brumnenwässer I, II und III, insbesondere auch beim Vergleich mit dem angeblich reinen Brunnenwasser V, keinen Augenblick daran gezweifelt werden, dass alle drei Brunnen durch die Abwässer der Zeche verunreinigt und infolgedessen für Trink- und häusliche Gebrauchszweeke unbrauchbar geworden waren.

Anders lagen indes die Verhältnisse bei dem Brunnen IV; bei ihm war die Menge der Chloride gegenüber den Brunnenwässern I, II und III nur gering, dagegen der Gehalt an Nitraten ein sehr beträchtlicher. Ebenso war aber auch der Schwefelsäuregehalt des Brunnens IV ein verhältnismäbig hoher und daher der Schluß berechtigt, daß der Brunnen IV zwar wahrscheinlich auch z. T. durch das Sickerwasser der Anschüttungen verunreinigt wurde, daß aber die wesentlichste Verunreinigung durch Zuflüsse aus mit menschlichen oder tierischen Abfallstoffen — die Düngergrube lag nur wenige Meter entfernt -- verunreinigten Bodenschichten herrührten. Der Brunnen IV hatte übrigens, wie aus der Analyse IV b hervorgeht, schon im Jabre 1896 eine ganz ähnliche Zusammensetzung. Die Zusanmensetzung eines reinen Kesselbrumnenwassers der dortigen Gegend veranschaulicht das Brunnenwasser VI, welches zwar anberordentlich hart, im übrigen aber nach der chemischen Analyse nicht verunreinigt war. Eine ähnliche Zusammensetzung wie das Wasser des Brunnens IV hatte auch das zwischen diesem und dem Brunnen II entnommene Grundwasser. Bei diesem Wasser, das in einem Garten entnommen war, mußte der hohe Gehalt an Nitraten höcbstwahrscheinlich auf eine starke Zuführung von Stalldünger und Jauche zurückgefübrt werden, wie sie ja bei Gartenländereien fast allgemein vorgenommen wird.

Der vorliegende Fall zeigt wieder recht deutlich, wie wichtig eine genaue chemische Analyse des Wassers und die Kenntnis der einschlägigen geologischen Verhältuisse zu sacbgemäßen Beurteilungen von Brunnen-Verunreinigungen sind.

Nach der Frühstückspause von 12-12//2 Uhr wird die Wahl des nächstjährigen Versammlungsortes vorgenommen und dafür Dresden bestimmt.

In Ergänzung seiner diesbezüglichen Mitteilungen ${ }^{1}$ ) führt darauf Dr. W. Rullma n n-München die zur Erkennung erhitzt gewesener Milch dienenden Reaktionen mit Wasserstoffsuperoxyd und alkoholischer Guajakharzlösung, sowie die Verfahren nach Schardinger und nach Storch vor, die vom Vortragenden als Zonenreaktionen ausgearbeitet wurden. Die Anwesenden hatten Gelegenheit, sich von der auBerordentlichen Schärfe und Sicherheit zu überzengen, mit der der Nachweis einer Erhitzung der Milch über $70^{\circ}$ oder von Mischungen roher und gekochter Milch geführt werden kann.

Hierauf folgte die Demonstration:

1) Diese Zeitschrift 1904, 7,81. 